\title{
Cognitive WSN transmission control for energy efficiency under WLAN coexistence
}

\author{
Ioannis Glaropoulos, Viktoria Fodor \\ KTH, Royal Institute of Technology \\ Stockholm, Sweden \\ Email: \{ioannisg,vfodor\}@kth.se
}

\author{
Loreto Pescosolido, Chiara Petrioli \\ University of Rome "La Sapienza" \\ Rome, Italy \\ Email: loreto@infocom.uniroma1.it,petrioli@di.uniroma1.it
}

\begin{abstract}
In this paper we design a cognitive access scheme for WSNs that coexist with WLANs, considering the problem of blind and hidden WLAN terminals. The cognitive access scheme relies on a-priory known WLAN idle time distribution functions and repeated channel measurements to optimize the size of the transmitted data unit and the transmission distance, such that the normalized transmission energy is minimized. We compare the proposed scheme with simple carrier sensing and random access solutions and show that the energy gain is significant under typical WLAN utilization values.
\end{abstract}

\section{INTRODUCTION}

Wireless LANs (WLANs) and wireless sensor networks (WSNs) operate often in the same geographic area and spectrum space. Due to the significant difference of the WLAN and WSN transmission powers, WLAN transmissions cause significant interference in the sensor network, making the sensor network communication unreliable, while the sensor network has negligible impact on the WLAN operation [1].

The goal of our work is to introduce cognitive capabilities in the WSN to decrease the negative effect of the co-existing WLAN. Specifically, we consider energy limited sensor networks, where the main goal of the cognitive operation is to increase energy efficiency and to prolong in this way the network lifetime. We define a cognitive access scheme that uses energy optimal packet size and transmission distance and applies repeated spectrum sensing to decide about transmission opportunities, utilizing a-priory known WLAN channel occupancy distributions. We build our work on the results of [2], where empirical WLAN channel occupancy models are defined, and on the results of [1] where the problem of blind WLAN terminals is considered in a scenario similar to ours, and the possibility of estimating the WLAN idle period distribution is demonstrated.

While the issue of energy efficient transmission in WSNs has been widely discussed [3], [4], even for coexisting networks [5], the effect of sensing and coordination impairments on the energy efficiency has not yet been addressed [6].

The rest of the paper is organized as follows. We describe the networking scenario in Section II, and the proposed cognitive access control in Section III, along with the model of the successful packet transmission and the optimization problem formulation. We evaluate the performance of our scheme in Section IV, and conclude the work in Section V.

\section{The Networking Scenario}

We consider a mesh IEEE 802.15.4 compliant WSN and a WLAN coexisting in the same geographic area, where the WSN operates on a narrow $3 \mathrm{MHz}$ band within the WLAN channel. Our goal is to introduce cognitive functionalities to the WSN channel access control and next hop selection, without significant modifications in the WSN protocol stack. To define the cognitive access scheme we take into account the main channel access characteristics of WLANs and WSNs.

The cognitive WSN scheme has two main building blocks:

- The packet size and the next hop distance are optimized based on the WLAN channel occupancy statistics and the sensing performance;

- An efficient sensing process is applied to predict whether there is sufficient WLAN idle time for the transmission of a WSN packet.

Since our goal is to decrease the energy consumption of the WSN nodes, we measure the performance of the proposed solution in terms of normalized energy cost, defined as the energy required to successfully transmit one unit of information over one unit of distance. That is, we do not impose strict constraints on the data delivery delay or on the network throughput, and do not take the WSN to WLAN interference into account, as its effect is negligible. Some assumptions are posed to simplify modeling. i) The WLAN user terminals are uniformly distributed around the WLAN access point, and all WLAN and WSN nodes are static. ii) Power and rate control are not applied in any of the networks. iii) The WSN load is very low, thus contention and in-network interference have a negligible impact on the WSN. While we aim at relaxing these assumptions in future work, we believe that they do not significantly affect the conclusions of the paper.

The performance of the proposed system depends on the WLAN channel occupancy, on WLAN interference and on the sensing performance, which are modeled as follows.

\section{A. WLAN Channel Occupancy Model}

Following the main ideas of [2], we consider a two-state semi-Markovian system to model the WLAN active (A) and idle (I) periods, parameterized with $f_{A}(t), f_{I}(t)$ respectively, with average values $\bar{T}_{A}, \bar{T}_{I}$ and steady state probabilities $p_{A}$, $p_{I}$. The function $f_{A}(t)$ corresponds to the transmission time 
of a single WLAN packet and is independent from the WLAN load. The idle time distribution is the linear combination of the back-off time $f_{I}^{(1)}(t)$ and WLAN inactivity period distributions $f_{I}^{(2)}(t)$, that is, $f_{I}(t)=p \cdot f_{I}^{(1)}(t)+(1-p) \cdot f_{I}^{(2)}(t)$, where $p$ denotes the percentage of idle periods due to back-off process and depends on the WLAN traffic pattern. The back-off time distribution is considered uniform and independent of the WLAN load. In this paper we assume that the back-off times are always shorter than the packet transmission times, which is typical in most cases. The length of the inactivity periods can be approximated with a generalized Pareto distribution, with parameters depending on the WLAN traffic pattern. Since the short back-off periods can not be used efficiently for WSN transmissions, the cognitive access control scheme should filter out these periods.

\section{B. Interference Modeling and Sensing Framework}

Sensors can successfully receive a packet, if the average experienced signal-to-noise-plus-interference ratio (SINR) is above a predefined power threshold, $\lambda$, which is the same for each sensor. We consider path-loss based channel model, which leads to a disc shaped interference region around each sensor. Since the path attenuation exponent is the same for the two coexisting networks, the radius of the interference region, $R_{\mathcal{I}}(r)$, is given -neglecting noise power - as $R_{\mathcal{I}}(r)=$ $r\left(\frac{\lambda P_{\mathrm{WLAN}}}{P_{\mathrm{WSN}}}\right)^{\frac{1}{\alpha}}$, where $r$ is the distance of the transmitting and receiving sensor, $\alpha$ denotes the path-loss exponent, $\lambda$ is the SINR bound and $P_{\mathrm{WSN}}, P_{\mathrm{WLAN}}$ denote the transmitting powers of the WSN and WLAN, respectively.

Since our goal is to extend existing WSN protocol stacks specifically 802.15.4 - with cognitive functionality, we build the sensing framework on sensing functions implemented in today's sensor nodes [7]. The sensors employ energy detection for a sensing period, $t_{s}$. After sensing, the spectrum is declared to be idle (I) or active (A) by comparing the measured energy to a tunable energy decision threshold $\gamma$. The probability of false alarm $p_{F A}$ depends on $\gamma$ and $t_{s}$, while the probability of missed signal detection depends on $P_{\mathrm{WLAN}}$ as well as on the distance $y$ between a WLAN transmitter and a receiving sensor, that is, $p_{M D}=p_{M D}(y)$. A detailed description of the performance of energy detection is presented in [8].

For the considered networking scenario we can approximate the location of the WLAN transmitters around the receiving sensor with a uniform distribution. Then the unconditioned probability of missed signal detection is $\overline{p_{M D}}=$ $\frac{1}{\pi R_{\max }^{2}} \int_{0}^{R_{\max }} p_{M D}(y) \cdot 2 \pi y d y$, where $R_{\max }$ denotes the maximum possible distance. Due to the large WLAN coverage area, $\mathcal{A}_{\text {WLAN }}$, and the limited spectrum sensing time, the probability of detecting WLAN transmitters far away is rather low, and is expected to affect the efficiency of the cognitive scheme.

\section{Cognitive Channel Access Control}

\section{A. Protocol Description}

We assume that WSN nodes follow a duty cycle and a slot synchronized MAC. At the beginning of the duty cycle each sensor performs spectrum sensing. The goal of spectrum

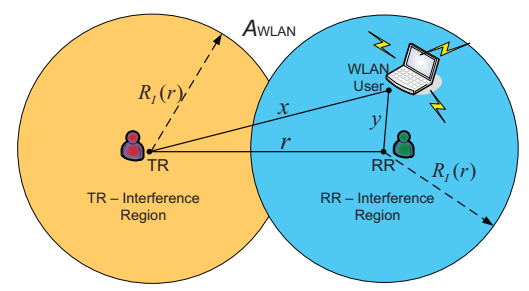

Fig. 1. The considered interference modeling scheme; TR-RR communication is successful as long as WLAN active users lie outside the interference regions of the pair of communicating sensors.

sensing in the cognitive system is twofold: to decide whether spectrum is idle and to evaluate, whether an idle period is due to WLAN back-off, or inactivity. To filter out the backoff periods, nodes perform repeated spectrum sensing with a time interval of $t_{\text {gap }}$, set as the maximum possible value of the back-off periods, given by $f_{I}^{(1)}$. That is, the length of the sensing window is $T_{s}=2 t_{s}+t_{\text {gap }}$. Since according to $f_{A}(t)$ active periods can not be shorter than $T_{s}$, nodes that sense idle spectrum at both sensing instances assume that the spectrum was idle in the entire time, and thus it is idle due to WLAN inactivity. Nodes with idle measurements remain awake to transmit (TR node) or to receive ( $\mathrm{RR}$ node) a packet. We consider two cases of MAC: TR nodes may transmit immediately after spectrum sensing, or may perform an RTS/CTS handshake, which adds a time overhead of $t_{\mathrm{hs}}$ in the WSN duty cycle. Nodes transit to sleep mode if at least one of the sensing instances showed the spectrum to be occupied.

Since the channel occupancy decision is based on local sensing information, the TR node may need to select an $\mathrm{RR}$ nearby, to ensure that the receiver experience a similar interference environment. Shorter transmission distances, though, imply that the energy efficiency of a multi-hop packet transmission from a source to a destination decreases (sensors transmit with fixed power and coding rate as often the case in practice). Similarly, selecting large packets may increase the energy efficiency due to the low control overhead, however, it decreases the probability that the WLAN idle period is long enough for successful transmission. Therefore, both the transmission distance and the packet size must be selected through careful optimization.

\section{B. Probabilistic Model of Successful Packet Transmission}

We derive an analytic model for the successful packet transmission and formulate the optimization problem along with a simple model for the energy cost of WSN communication.

Let us assume that the TR discovers an opportunity for transmission after a pair of idle spectrum measurements. A packet will be successfully transmitted from the TR to the RR if: 1) the RR node is active as well as result of a pair of idle spectrum measurements; 2) there is no transmitting WLAN user inside the interference regions of the TR and RR during handshake time if handshake is supported, and in the interference region of RR otherwise; 3) no WLAN user inside the interference region of RR starts transmitting at any time 
during the transmission of the WSN packet. Note, that due to our assumption on low WSN load, TR nodes always get transmission opportunity if requirements 1 and 2 are met.

We proceed with the derivation of the probability of successful packet transmission as follows. First we derive the idle and active spectrum status probabilities, conditioned on the TR idle spectrum measurements. We then determine the distribution of the interference-free time available for TR-RR communication. The probability of successful packet transmission is then expressed with respect to these terms and the conditional probability of the RR node being active.

We denote the TR idle sensing events during the first and the second measurement as $\hat{I}_{1}$ and $\hat{I}_{2}$, and define by $S_{i}^{\left(x_{i}, y_{i}\right)}$ the status of the spectrum at distances $x_{i}, y_{i}$ from the TR and RR sensors, respectively, during the $i$-th measurement, with $S_{i}^{\left(x_{i}, y_{i}\right)} \in \mathcal{S}=\{\mathrm{A}, \mathrm{I}\}$, where $A, I$ denote "active" and "idle" spectrum status, $i=1,2$ and $\left(x_{i}, y_{i}\right) \in \mathcal{X} \times \mathcal{Y}=\mathcal{A}_{\text {WLAN }} . S_{i}$ defines the status of the spectrum indexed by the measurement time, regardless of spatial dependence. For an arbitrary point in time and space we omit both indexes. The densities $f_{X}(x)$, $f_{Y}(y)$ and $f_{X Y \mid r}(x, y)$ denote the spatial distribution of the WLAN users with respect to the TR and RR separated by distance $r$ (Figure 1) and are derived with basic geometry.

1) Spectrum status: To calculate $\left(S_{1}^{y_{1}}, S_{2}^{y_{2}}\right) \mid \hat{I}_{1}, \hat{I}_{2}$ for all $x \in \mathcal{X}$ with $S_{1}, S_{2} \in \mathcal{S}$ we use the decomposition:

$$
P\left\{\left(S_{1}^{y_{1}}, S_{2}^{y_{2}}\right) \mid \hat{I}_{1}, \hat{I}_{2}\right\}=P\left\{S_{1}^{y_{1}} \mid \hat{I}_{1}\right\} P\left\{S_{2}^{y_{2}} \mid S_{1}^{y_{1}}, \hat{I}_{2}\right\} .
$$

We compute the first factor of (1), $P\left\{S_{1}^{y_{1}} \mid I_{1}\right\}$, by applying the Bayes rule as:

$$
\begin{aligned}
& P\left\{I_{1} \mid \hat{I}_{1}\right\}=\frac{p_{I}\left(1-p_{F A}\right)}{p_{I}\left(1-p_{F A}\right)+p_{A} \overline{p_{M D}}}, \\
& P\left\{A_{1}^{y_{1}} \mid \hat{I}_{1}\right\}=\frac{p_{A} \int_{\mathcal{X}} p_{M D}(x) f_{X Y \mid r}\left(x, y_{1}\right) d x}{p_{I}\left(1-p_{F A}\right)+p_{A} \overline{p_{M D}}} .
\end{aligned}
$$

For the derivation of the second factor of (1) we first need to determine the updated status distribution conditioned on the status during the first measurement, $S_{2}^{y_{2}} \mid S_{1}^{y_{1}}$. For $S_{1}=I$, (Figure 2.a,c), we obtain:

$$
\begin{aligned}
& p_{I_{2} \mid I_{1}}=P\left\{\text { Remaining idle time }>t_{\text {gap }}\right\}=\bar{F}_{R_{I}}\left(t_{\text {gap }}\right), \\
& p_{A_{2}^{y_{2} \mid I_{1}}}=\left[1-p_{I_{2} \mid I_{1}}\right] f_{Y}\left(y_{2}\right) .
\end{aligned}
$$

where $f_{R_{S}}(t)=\int_{t}^{\infty} f_{S}(z) / z d z, S \in\{A, I\}$ assuming that the first measurement is uniformly distributed within the active or idle period. For $S_{1}=A, S_{2}=I$, (Figure 2.b) we obtain:

$$
p_{I_{2} \mid A_{1}^{y_{1}}}=\int_{0}^{t_{\text {gap }}} \bar{F}_{I}\left(t_{\text {gap }}-z\right) f_{R_{A}}(z) d z, \forall y_{1} .
$$

Finally, for $S_{2}=A$ we need to distinguish between a continuous active period and the case when there is a short idle period between the two sensing measurements (Figure 2.d):

$p_{A_{2}^{y_{2}} \mid A_{1}^{y_{1}}}=\bar{F}_{R_{A}}\left(t_{\text {gap }}\right) \delta\left(y_{1}\right)+\left(1-p_{I_{2} \mid A_{1}^{y_{1}}}-\bar{F}_{R_{A}}\left(t_{\text {gap }}\right)\right) f_{Y}\left(y_{2}\right)$.

We can now express the second factor of (1). For $S_{2}=I$ :

$$
\begin{aligned}
& P\left\{I_{2} \mid I_{1}, \hat{I}_{2}\right\}=\frac{p_{I_{2} \mid I_{1}}\left(1-p_{F A}\right)}{p_{I_{2} \mid I_{1}}\left(1-p_{F A}\right)+\overline{p_{M D}} p_{A_{2} \mid I_{1}}}, \\
& P\left\{I_{2} \mid A_{1}^{y_{1}}, \hat{I}_{2}\right\}= \\
& =\frac{p_{I_{2} \mid A_{1}}\left(1-p_{F A}\right)}{p_{I_{2} \mid A_{1}}\left(1-p_{F A}\right)+\int_{\mathcal{Y}} p_{A_{2}}^{y_{2}} \mid A_{1}^{y_{1}} \int_{\mathcal{X}} p_{M D}(x) f_{X \mid y_{2}}\left(x, y_{2}\right) d x d y} .
\end{aligned}
$$

Similarly, for $S_{1}=\left\{I_{1}, A_{1}^{y_{1}}\right\}, S_{2}=A$, we obtain:

$$
\begin{aligned}
P & \left\{A_{2}^{y_{2}} \mid S_{1}, \hat{I}_{2}\right\}= \\
& =\frac{p_{A_{2}^{y_{2}} \mid S_{1}} \int_{X} p_{M D}(x) f_{X \mid y_{2}}\left(x, y_{2}\right) d x}{p_{I_{2} \mid S_{1}}\left(1-p_{F A}\right)+\int_{\mathcal{Y}} p_{A_{2}^{y_{2}} \mid S_{1}} \int_{\mathcal{X}} p_{M D}(x) f_{X \mid y_{2}}\left(x, y_{2}\right) d x d y} .
\end{aligned}
$$
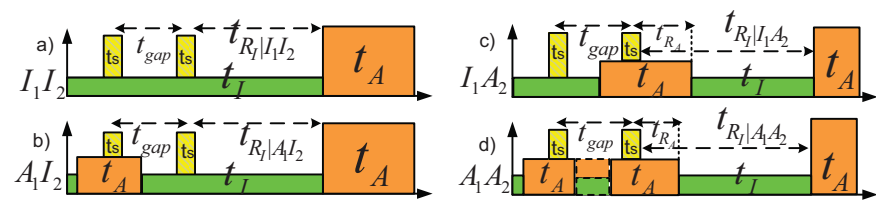

Fig. 2. Spectrum status cases at the time of the sensing measurements.

2) Remaining TR transmission time: The remaining transmission time distribution needs to be derived for each spectrum status $\left(S_{1}, S_{2}\right)$. If the spectrum was idle during both sensing measurements, the WLAN can be considered as inactive, as explained in Section III. Considering that the beginning of the sensing period $T_{s}=2 t_{s}+t_{\text {gap }} \approx t_{\text {gap }}{ }^{1}$ is uniformly distributed inside the current WLAN inactive period, the remaining idle time has the following density function:

$$
f_{R_{I} \mid I_{1}, I_{2}}(t)=\int_{t+t_{\text {gap }}}^{\infty} \frac{1}{z} f_{I}^{(2)}(z) d z / \bar{F}_{R_{I}}\left(t_{\text {gap }}\right) .
$$

The distribution of the remaining idle time is, however, different when one or both spectrum measurements have been erroneous, since now the spectrum may be or become idle due to back-off as well. Consider first, that the spectrum status was $\left(A_{1}, I_{2}\right)$, so the first measurement was erroneous. Since the spectrum is idle during the second measurement, the transition from active to idle occurs sometime before $t_{\text {gap }}$. Consequently, the remaining idle time after the second measurement has the following density:

$$
f_{R_{I} \mid A_{1}, I_{2}}(t)=\frac{1}{F_{R_{A}}\left(t_{\text {gap }}\right)} \int_{0}^{t_{\text {gap }}} f_{R_{A}}(z) \frac{f_{I}\left(t+t_{\text {gap }}-x\right)}{\bar{F}_{I}\left(t_{\text {gap }}-z\right)} d z .
$$

For $S_{2}=A$ the spectrum is active after the second measurement, so successful TR-RR communication requires that the WLAN source lies outsider the interference region(s). If this requirement is satisfied, the TR can reliably transmit within the remaining active spectrum period, and in the idle period that follows immediately.

For $\left(I_{1}, A_{2}\right)$ (Figure 2.c) the distribution of the remaining idle time after the second measurement is:

$$
f_{R_{I} \mid I_{1}, A_{2}}(t)=\frac{1}{F_{R_{I}}\left(t_{\text {gap }}\right)} \int_{0}^{t_{\text {gap }}} f_{R_{I}}(z) \frac{f_{\text {cycle }}\left(t+t_{\text {gap }}-z\right)}{\bar{F}_{\text {cycle }}\left(t_{\text {gap }}-z\right)} d z
$$

where $f_{\text {cycle }}(t)=f_{\mathrm{A}}(t) * f_{I}(t)$ is the density function of the duration of a WLAN cycle defined as a pair of successive active and idle periods.

We consider finally the case $\left(A_{1}, A_{2}\right)$ (Figure 2,d), when the spectrum is indeed active despite the idle measurements. For the subcase of a continuous active period between the two measurements the remaining transmission time is:

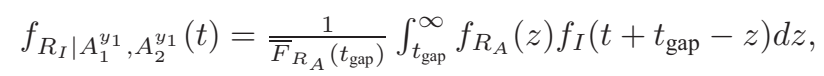

\footnotetext{
${ }^{1}$ The time gap between successive sensing measurements is at least an order of magnitude larger than the sensing measurement periods
} 
while in the case of a short idle period:

$$
\begin{aligned}
& f_{R_{I} \mid A_{1}^{y_{1}}, A_{2}^{y_{2}}}(t)=\frac{1}{\bar{F}_{R_{A}}\left(t_{\text {gap }}\right)} . \\
& \cdot \int_{0}^{t_{\text {gap }}} f_{R_{A}}\left(z_{1}\right) \frac{\int_{0}^{t_{\text {gap }}-z_{1}} f_{I}\left(z_{2}\right)}{F_{I}\left(t_{\text {gap }}-z_{1}\right)} \cdot \frac{f_{\text {cycle }}\left(t+t_{\text {gap }}-z_{1}-z_{2}\right)}{F_{A}\left(t_{\text {gap }}-z_{1}-z_{2}\right)} d z_{1} d z_{2} \text {. }
\end{aligned}
$$

(8)-(12) give the remaining transmission time distribution after spectrum sensing. However, the expiration of this time period does not affect the TR-RR communication, as long as the activated WLAN sources lie outside the RR interference region. Consequently, a geometric number of successive WLAN cycle periods must be added to the remaining time computed above. The density function of this additional interference-free time $f_{\tilde{I}}(t)$ can easily be calculated in the Laplace domain. The total remaining time available for WSN communication is finally:

$$
f_{T_{R_{I}} \mid S_{1}, S_{2}}(t)=f_{R_{I} \mid S_{1}, S_{2}}(t) * f_{\tilde{I}}(t), \forall S_{1}, S_{2} \in \mathcal{S} .
$$

3) The probability of successful transmission without/with handshake: Let us consider the case without handshake first. We define the event tuning $\mathcal{T}$, when the TR and RR nodes start to communicate successfully. As handshake is not implemented, packet transmission starts immediately after the sensing period and successful packet transmission requires tuning and adequate idle time.

The tuning probability under different spectrum status is:

$$
\begin{aligned}
& P\left\{\mathcal{T}, S_{1}, I_{2} \mid \hat{I}_{1}, \hat{I}_{2}\right\}= \\
& \quad \iint_{\mathcal{Y}^{2}} P\left\{\left(\hat{I}_{1}^{(R)}, \hat{I}_{2}^{(R)}\right) \mid S_{1}, I_{2}\right\} P\left\{S_{1}, I_{2} \mid \hat{I}_{1}, \hat{I}_{2}\right\} d y_{1} d y_{2}, \\
& P\left\{\mathcal{T}, S_{1}, A_{2}^{y_{2}} \mid \hat{I}_{1}, \hat{I}_{2}\right\}= \\
& \quad \int_{\mathcal{Y}} \int_{\mathcal{Y} \backslash\left\{y: y \geq R_{\mathcal{I}}(r)\right\}} P\left\{\left(\hat{I}_{1}^{(R)}, \hat{I}_{2}^{(R)}\right) \mid S_{1}, A_{2}^{y_{2}}\right\} . \\
& \quad \cdot P\left\{S_{1}, A_{2}^{y_{2}} \mid \hat{I}_{1}, \hat{I}_{2}\right\} d y_{1} d y_{2},
\end{aligned}
$$

where $P\left\{\left(\hat{I}_{1}^{(R)}, \hat{I}_{2}^{(R)}\right) \mid S_{1}, S_{2}\right\}$ is the probability of idle measurements at the receiver under given spectrum status, and can be calculated from $p_{F A}$ and $p_{M D}(y)$.

Finally, the probability of successful packet transmission without handshake is calculated from (13) and (14) by averaging over the spectrum status:

$$
\begin{aligned}
& P\{\text { Successful Transmission } \mid r, t\}= \\
& \sum_{\mathcal{S}^{2}} \bar{F}_{T_{R_{I}} \mid S_{1}, S_{2}}(t) \cdot P\left\{\mathcal{T}, S_{1}, S_{2} \mid \hat{I}_{1}, \hat{I}_{2}\right\} .
\end{aligned}
$$

When handshake is adopted, the successful RTS/CTS handshake (HS) offers the a-posteriori knowledge of both successful tuning and user-free TR interference region, to the cost of a time overhead equal to $t_{\mathrm{hs}}$. Therefore, the probability of successful transmission becomes:

$$
\begin{aligned}
& P\{\text { Successful Transmission with HS } \mid r, t\}= \\
& =\sum_{\mathcal{S}^{2}} \bar{F}_{T_{R_{I}} \mid S_{1}, S_{2}}\left(t+t_{\mathrm{hs}}\right) P\left\{S_{1}, S_{2} \mid \hat{I}_{1}, \hat{I}_{2}, \mathcal{T}_{H S}\right\} .
\end{aligned}
$$

where the status probabilities conditioned on tuning are given in (17). The a priori tuning probability is computed as:

$$
P\left\{\mathcal{T}_{H S}\right\}=\sum_{\mathcal{S}^{2}} P\left\{\mathcal{T}, S_{1}, S_{2} \mid \hat{I}_{1}, \hat{I}_{2}\right\},
$$

with the additional constraint $x \geq R_{\mathcal{I}}(r)$ applied directly in the integrals of the nominator of (7).
TABLE I

PARAMETER SET FOR THE PERFORMANCE EVALUATION.

\begin{tabular}{|c|c|}
\hline WLAN Tx-Power $\left(P_{\text {WLAN }}\right): 15 \mathrm{dBm}$ & WSN Tx-Power $\left(P_{\mathrm{WSN}}\right):-3 \mathrm{dBm}$ \\
Path-Loss $(\alpha): 4$ & SINR $(\lambda): 3 \mathrm{~dB}$ \\
WSN Power Cons. $(P): 35 \mathrm{~mW}$ & Tx-Rate $(R): 250 \mathrm{kbps}$ \\
Sensing Time $\left(t_{s}\right): 16 \mu \mathrm{s}$ & inter-sens. period $\left(t_{\text {gap }}\right): 0.5 \mathrm{~ms}$ \\
Handshake overhead $\left(t_{\mathrm{hs}}\right): 64 \mu \mathrm{s}$ & WSN pckt. overhead $\left(L_{\mathrm{hd}}\right): 17 \mathrm{Bytes}$ \\
\hline
\end{tabular}

\section{Energy cost model and optimization for energy efficiency}

We consider a simple energy model with constant power consumption, $P$, at the sensors at all active states (sensing, transmission and reception), while the power spent at the sleep state is negligible, and assume that transmission attempts are stochastically independent, due to the long WSN duty cycle. The transmission duration of a packet of length $L$ is $t_{L}=\left(L+L_{\mathrm{hd}}\right) / R$, where $R$ is the WSN transmission rate and $L_{\mathrm{hd}}$ denotes the header protocol overhead. When handshake is not used, the expected energy cost for the transmission to a receiver at distance $r$ can be modeled as:

$$
E(r, L)=\frac{P \cdot\left(t_{L}+2 t_{s}\right)}{P\left\{\text { Successful Transmission } \mid r, t_{L}\right\}} .
$$

With handshake a number of tuning and transmission attempts may be needed for successful packet transmission, and the energy consumption must also include the handshake time $t_{h s}$. The expected energy cost for tuning is $E_{\mathcal{T}}(r)=\frac{P \cdot\left(t_{h s}+2 t_{s}\right)}{P\left\{\mathcal{T}_{H S}\right\}}$, and the total cost for packet transmission becomes:

$$
E_{H S}(r, L)=\frac{E_{\mathcal{T}}(r)+P \cdot t_{L}}{P\left\{\text { Successful Transmission with HS } \mid r, t_{L}\right\}} .
$$

Given the energy cost models, we optimize parameters $L, r$ and detection threshold $\gamma$, such that the energy cost normalized with $L \cdot r$ is minimized.

\section{Performance Evaluation}

We evaluate the performance of the proposed solution considering the normalized energy cost under different WLAN loads and traffic distribution. For comparison, we have modeled the energy consumption of a CSMA-based MAC scheme without repeated sensing and that of a random channel access scheme, both under optimized packet size and transmission distance. These models are similar to the presented ones, and are not shown due to space limitation. Both cases with or without handshake functionality are presented for comparative evaluation. Following [2], [9] we consider $f_{A} \sim \mathrm{U}(0.5,1.5 \mathrm{~ms}), f_{I}^{(1)} \sim \mathrm{U}(10 \mu \mathrm{s}, 0.5 \mathrm{~ms})$ and $f_{I}^{(2)} \sim$ $\operatorname{GPareto}(\mu, \sigma, \xi)$ with location parameter $\mu=0$, scale parameter $\xi=-0.3095$ and shape parameter $\sigma$ determined by the desired WLAN load and $p$. The rest of the parameters are summarized in Table I.

Figures 3 and 4 depict the performance of the proposed cognitive MAC compared to CSMA and random access schemes, for both cases with and without handshake functionality, as a function of WLAN spectrum load. In Figure 3 the load is increased by increasing the percentage $p$ of back-off periods, while keeping the inactivity period distribution $f_{I}^{(2)}$ constant, 


$$
P\left\{S_{1}, S_{2} \mid \hat{I}_{1}, \hat{I}_{2}, \mathcal{T}_{H S}\right\}=\frac{\int_{\mathcal{Y}} \int_{\mathcal{X} Y \backslash\left\{x_{2}, y_{2} \leq R_{\mathcal{I}}(r)\right\}} P\left\{\mathcal{T}_{H S} \mid S_{1}^{y_{1}}, S_{2}^{\left(x_{2}, y_{2}\right)}\right\} P\left\{S_{1}^{\left(y_{1}\right.}, S_{2}^{\left(x_{2}, y_{2}\right)} \mid \hat{I}_{1}, \hat{I}_{2}\right\} d x_{2} d y_{1} d y_{2}}{\sum_{\left(S_{1}, S_{2}\right) \in \mathcal{S}^{2}} \int_{\mathcal{Y}} \int_{\mathcal{X} \mathcal{Y} \backslash\left\{x_{2}, y_{2} \leq R_{\mathcal{I}}(r)\right\}} P\left\{\mathcal{T}_{H S} \mid S_{1}^{\left(y_{1}\right.}, S_{2}^{\left(x_{2}, y_{2}\right)}\right\} P\left\{S_{1}^{y_{1}}, S_{2}^{\left(x_{2}, y_{2}\right)} \mid \hat{I}_{1}, \hat{I}_{2}\right\} d x_{2} d y_{1} d y_{2}}
$$
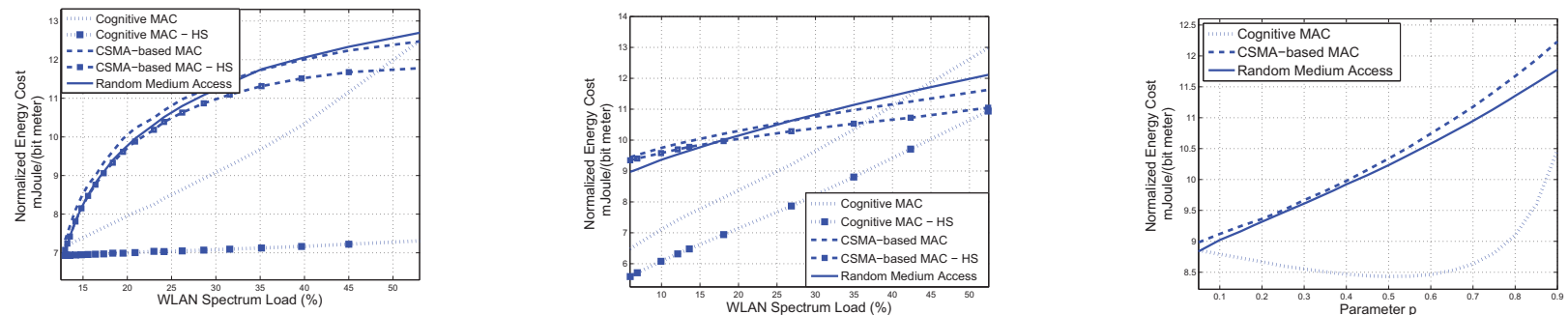

Fig. 3. Energy Cost with respect to WLAN Load, under different percentages of back-off idle periods in $f_{I}(t), \overline{T_{I}^{(2)}}=3.5 \mathrm{~ms}$
Fig. 4. Energy Cost with respect to WLAN Load, under decreasing expected lengths of WLAN users' inactivity periods, $p=0.5$
Fig 5 Energy Cost with respect to the parameter $p$ that shapes the mixed idle distribution. Fixed WLAN Load $=22 \%$ while in Figure 4 we keep $p$ constant, and decrease the average length of the inactivity period. We can see that the increase of the energy consumption is significantly different in the two cases, that is, the average WLAN load is not enough to characterize the WSN efficiency. For example, in Figure 3 when low load means low probability of back-off periods the cognitive scheme does not increase the efficiency, while in Figure 4, when half of the the idle periods are back-offs even under low load, the filtering of the back-off periods in the cognitive scheme leads to significant gain. We observe that the additional energy cost and sensing time can make the CSMA and cognitive schemes inefficient in low and high WLAN load regimes. In both cases, however, the cognitive scheme outperforms significantly the others for typical, moderate WLAN load values. Handshaking increases the efficiency in particular when the load is increased under constant inactive period distribution when the load hardly affects the energy efficiency. Figure 5 compares the performance of the three systems for constant WLAN load, tuned by the parameter $p$, simultaneously increasing the average length of the inactive periods. The cognitive system clearly outperforms the other systems in all but the marginal cases, when it also gives a robust performance, independently of the traffic characteristics. Consequently, an adaptive medium access scheme is necessary to cover all range of possible WLAN loads. Finally, in Figure 6 we evaluate the effect of sensing performance for the scenario of Figure 4, by increasing the channel detection time and comparing the results to the perfect sensing case. We can conclude, that the energy consumption of the cognitive scheme is close to the lower bound for acceptable sensing time values.

\section{CONCLUSION}

In this paper we have proposed a cognitive channel access scheme for WSNs to minimize the normalized transmission energy cost under WLAN interference, specifically considering the limited sensing capability of the sensor nodes. We have

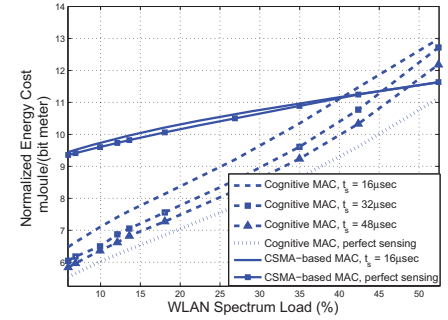

Fig. 6. Energy Cost with respect to WLAN Load for various channel detection times, with perfect sensing schemes for comparison.

shown that the proposed solution achieves significant performance gain compared to traditional channel access solutions under typical WLAN load values. The work will be extended by the design of the complete cognitive cycle, including the in-network estimation of WLAN channel occupancy functions.

\section{REFERENCES}

[1] J. Huang, G. Xing, G. Zhou, and R. Zhou. Beyond co-existence; exploiting wifi white space for zigbee performance assurance. In IEEE International Conference on Network Protocols, 2010.

[2] S. Geirhofer, L. Tong, and B. M. Sadler. Cognitive medium access: Constraining interference based on experimental models. IEEE Selected Areas in Communications, 26(1), 2008.

[3] J. N. Al-karaki and A. E. Kamal. Routing techniques in wireless sensor networks: A survey. IEEE Wireless Communications, 11, 2004.

[4] T. Watteyne A. Bachir, M. Dohler and K.K. Leung. Mac essentials for wireless sensor networks. IEEE Communications Surveys Tutorials, 2010

[5] S. Gao, L. Qian, and D.R. Vaman. Distributed energy efficient spectrum access in wireless cognitive radio sensor networks. In IEEE Wireless Communications and Networking Conference, 2008.

[6] O. Karli O. Akan and O. Ergul. Cognitive radio sensor networks. IEEE Network, 23(4), 2009.

[7] Chipson Products from Texas Instruments. 2.4ghz ieee 802.15.4/ zigbeeready rf transceiver. Technical report, 2008.

[8] V. Fodor, I. Glaropoulos, and L. Pescosolido. Detecting low-power primary signals via distributed sensing to support opportunistic spectrum access. In IEEE International Conference on Communications, June 2009.

[9] H. F. Campos, M. Karaliopoulos, M. Papadopouli, and H. Shen. Spatiotemporal modeling of traffic workload in a campus wlan. In WICON '06, 2006. 